\title{
Subadditive functions and partial converses of Minkowski's and Mulholland's inequalities
}

\author{
by \\ Janusz Matkowski (Bielsko-Biała) and \\ Tadeusz Świąt kowski (Łódź)
}

\begin{abstract}
Let $\phi$ be an arbitrary bijection of $\mathbb{R}_{+}$. We prove that if the two-place function $\phi^{-1}[\phi(s)+\phi(t)]$ is subadditive in $\mathbb{R}_{+}^{2}$ then $\phi$ must be a convex homeomorphism of $\mathbb{R}_{+}$. This is a partial converse of Mulholland's inequality. Some new properties of subadditive bijections of $\mathbb{R}_{+}$are also given. We apply the above results to obtain several converses of Minkowski's inequality.
\end{abstract}

Introduction. Throughout this paper $\mathbb{R}, \mathbb{R}_{+}$, and $\mathbb{N}$ will stand respectively for the set of reals, nonnegative reals, and positive integers.

Every function $f: \mathbb{R}_{+} \rightarrow \mathbb{R}$ satisfying the inequality

$$
f(s+t) \leq f(s)+f(t) \quad(s, t \geq 0)
$$

is said to be subadditive. If the inequality is reversed the function is termed superadditive.

In our recent paper [4] we have proved the following

Theorem 1. If $f: \mathbb{R}_{+} \rightarrow \mathbb{R}_{+}$is subadditive, right-continuous at 0 and bijective then $f$ is a homeomorphism of $\mathbb{R}_{+}$.

In Section 1 we consider the two-place function $\mathbf{p}_{\phi}: \mathbb{R}_{+}^{2} \rightarrow \mathbb{R}_{+}$given by the formula $\mathbf{p}_{\phi}(s, t):=\phi^{-1}[\phi(s)+\phi(t)]$ where $\phi: \mathbb{R}_{+} \rightarrow \mathbb{R}_{+}$is an arbitrary bijection. Using Theorem 1 we prove that if $\mathbf{p}_{\phi}$ is subadditive in $\mathbb{R}_{+}^{2}$ then $\phi$ is a convex homeomorphism of $\mathbb{R}_{+}$. This is a partial converse of Mulholland's criterion of subadditivity of the functional $\mathbf{p}_{\phi}$ (cf. H. P. Mulholland

1991 Mathematics Subject Classification: Primary 26D15, 46E30, 39C05; Secondary 26A51, 26A18.

Key words and phrases: subadditive function, homeomorphisms of $\mathbb{R}_{+}$, Mulholland's inequality, convex function, iteration, measure space, the converse of Minkowski's inequality. 
[6], also M. Kuczma [2], p. 201, Theorem 1). In Section 2 we apply this result to improve some converses of Minkowski's inequality for $\mathbf{l}_{2}^{p}$-norm.

In [5] we have shown that in Theorem 1 the right continuity at 0 cannot be replaced by a weaker assumption of boundedness in a neighbourhood of 0. However, in Section 3 of the present paper we prove among other things that if $\phi$ is a bijection of $\mathbb{R}_{+}$such that $\phi^{-1}$ and, for some positive integer $n>1$, the function $\phi \circ\left(n \phi^{-1}\right)$ are subadditive and $\phi^{-1}$ is bounded in a neighbourhood of 0 then $\phi$ is a homeomorphism of $\mathbb{R}_{+}$. This result together with Theorem 1 as well as the main result of Section 1 permit us (Section 4) to prove some new converses of Minkowski's inequality for the $\mathbf{L}^{p}$-norm. One of these results (Theorem 8) reads as follows. Let $(\Omega, \Sigma, \mu)$ be a measure space with at least three sets $A, B, C \in \Sigma$ such that

$$
0<\mu(A)<1, \quad \mu(B)=\mu(C)=1, \quad B \cap C=\emptyset .
$$

If $\phi: \mathbb{R}_{+} \rightarrow \mathbb{R}_{+}$is a bijection such that $\phi(0)=0$ and for all nonnegative $\mu$-integrable step functions $x, y$,

$$
\phi^{-1}\left(\int_{\Omega} \phi \circ|x+y| d \mu\right) \leq \phi^{-1}\left(\int_{\Omega} \phi \circ|x| d \mu\right)+\phi^{-1}\left(\int_{\Omega} \phi \circ|y| d \mu\right)
$$

then, without any regularity conditions, $\phi(t)=\phi(1) t^{p}(t \geq 0)$ for some $p \geq 1$.

1. Subadditive functions of the form $\phi^{-1}[\phi(s)+\phi(t)]$ and a partial converse of Mulholland's inequality. For an arbitrary bijection $\phi$ : $\mathbb{R}_{+} \rightarrow \mathbb{R}_{+}$the two-place function $\mathbf{p}_{\phi}: \mathbb{R}_{+}^{2} \rightarrow \mathbb{R}_{+}$given by the formula

$$
\mathbf{p}_{\phi}(x):=\phi^{-1}\left[\phi\left(x_{1}\right)+\phi\left(x_{2}\right)\right], \quad x=\left(x_{1}, x_{2}\right),
$$

is well defined. Functions of this form are known to be solutions of the associativity functional equation (cf. J. Aczél [1], p. 253). Moreover, if $\phi(t)=\phi(1) t^{p}, p \geq 1$, then, in view of Minkowski's inequality, $\mathbf{p}_{\phi}$ is subadditive. This classical fact has been generalized by H. P. Mulholland [6] (cf. also M. Kuczma [2], p. 198), who proved the following criterion of subadditivity for $\mathbf{p}_{\phi}$.

Mulholland's INEQUaLity. If $\phi: \mathbb{R}_{+} \rightarrow \mathbb{R}_{+}$is a convex homeomorphism of $\mathbb{R}_{+}$such that $\log \circ \phi \circ \exp$ is convex in $\mathbb{R}$ then the functional $\mathbf{p}_{\phi}$ is subadditive in $\mathbb{R}_{+}^{2}$.

Using Theorem 1, we prove a partial converse.

Theorem 2. Let $\phi: \mathbb{R}_{+} \rightarrow \mathbb{R}_{+}$be an arbitrary bijection of $\mathbb{R}_{+}$. If $\mathbf{p}_{\phi}$ is subadditive in $\mathbb{R}_{+}^{2}$ then $\phi$ is a convex homeomorphism of $\mathbb{R}_{+}$.

Pr o of. Writing out the subadditivity of $\mathbf{p}_{\phi}$ we have 
(1)

$$
\begin{aligned}
\phi^{-1}\left[\phi\left(x_{1}+y_{1}\right)+\phi\left(x_{2}\right.\right. & \left.\left.+y_{2}\right)\right] \\
& \leq \phi^{-1}\left[\phi\left(x_{1}\right)+\phi\left(x_{2}\right)\right]+\phi^{-1}\left[\phi\left(y_{1}\right)+\phi\left(y_{2}\right)\right]
\end{aligned}
$$

for all $x_{1}, x_{2}, y_{1}, y_{2} \geq 0$. Setting here $x_{1}=x_{2}:=s, y_{1}=y_{2}:=t$, we get

$$
\phi^{-1}[2 \phi(s+t)] \leq \phi^{-1}[2 \phi(s)]+\phi^{-1}[2 \phi(t)], \quad s, t \geq 0,
$$

which means that the function $f:=\phi^{-1} \circ(2 \phi)$ is subadditive in $\mathbb{R}_{+}$. Since $f$ maps $\mathbb{R}_{+}$onto $\mathbb{R}_{+}$, there exists a $t_{0} \geq 0$ such that $f\left(t_{0}\right)=0$. From (2) we have $f\left(2 t_{0}\right) \leq 2 f\left(t_{0}\right)=0$ and, consequently, $f\left(2 t_{0}\right)=f\left(t_{0}\right)$. Since $f$ is one-to-one it follows that $t_{0}=0$. Hence we get $f(0)=\phi^{-1}[2 \phi(0)]=0$, which implies that $\phi(0)=0$. Therefore, substituting $x_{1}:=s, x_{2}=y_{1}:=0$, $y_{2}:=t$ in (1), we get

$$
\phi^{-1}[\phi(s)+\phi(t)] \leq s+t, \quad s, t \geq 0 .
$$

In particular, $\phi^{-1}[2 \phi(t)] \leq 2 t(t \geq 0)$. This proves that the function $f=$ $\phi^{-1} \circ(2 \phi)$ is continuous at 0 . In view of Theorem $1, f$ is a homeomorphism of $\mathbb{R}_{+}$.

Substituting $x_{1}=y_{2}:=s, x_{2}=y_{1}:=t$ in (1) we get

$$
f(s+t) \leq 2 \phi^{-1}[\phi(s)+\phi(t)], \quad s, t \geq 0 .
$$

As $f$ is strictly increasing, it follows that

$$
f(t)<2 \phi^{-1}[\phi(s)+\phi(t)], \quad s, t>0 .
$$

Since $f(t)=t$ if and only if $t=0$ we have either

(a) $f(t)<t$ for all $t>0$, or

(b) $f(t)>t$ for all $t>0$.

We are going to show that case (a) cannot occur. To this end denote by $f^{n}$ the $n$th iterate of $f$. In case (a) the sequence $\left(f^{n}(t)\right)_{n=1}^{\infty}$ would be strictly decreasing for every $t>0$. Therefore, since $f^{n}(t)=\phi^{-1}\left[2^{n} \phi(t)\right]$, we would have from (4),

$$
\phi^{-1}\left[2^{n} \phi(t)\right]<2 \phi^{-1}[\phi(s)+\phi(t)], \quad s, t>0, n \in \mathbb{N} .
$$

Replacing $t$ by $\phi^{-1}\left[2^{-n} \phi(t)\right]$ we hence obtain

$$
t<2 \phi^{-1}\left[\phi(s)+2^{-n} \phi(t)\right], \quad s, t>0, n \in \mathbb{N} .
$$

Fix $t>0$. For $n \in \mathbb{N}$ sufficiently large we clearly have $\phi\left(2^{-1} t\right)-2^{-n} \phi(t)>0$. Substituting in the above inequality

$$
s:=\phi^{-1}\left[\phi\left(2^{-1} t\right)-2^{-n} \phi(t)\right]
$$

we get $t<t$, which is a contradiction.

This proves that $f$ satisfies inequality (b). 
Now we are in a position to prove that $\phi^{-1}$ is continuous at 0 . From (4) and (b) we have

$$
t<2 \phi^{-1}[\phi(s)+\phi(t)], \quad s, t>0,
$$

or, equivalently,

$$
\phi^{-1}(t)<2 \phi^{-1}(s+t), \quad s, t>0 .
$$

If $\phi^{-1}$ were discontinuous at 0 then there would exist an $\varepsilon>0$ and a sequence of positive reals $\left(t_{n}\right)_{n=1}^{\infty}$ such that

$$
\lim _{n \rightarrow \infty} t_{n}=0 \text { and } 2 \varepsilon \leq \phi^{-1}\left(t_{n}\right), \quad n \in \mathbb{N} .
$$

Hence, setting $t:=t_{n}$ in (5), we obtain

$$
\varepsilon \leq \phi^{-1}\left(s+t_{n}\right), \quad s>0, n \in \mathbb{N} .
$$

This inequality and the relation $\lim _{n \rightarrow \infty} t_{n}=0$ imply that

$$
\phi^{-1}[(0, \infty)]=\bigcup_{n=1}^{\infty} \phi^{-1}\left[\left(t_{n}, \infty\right)\right] \subseteq \bigcup_{n=1}^{\infty}[\varepsilon, \infty)=[\varepsilon, \infty),
$$

which contradicts the bijectivity of $\phi$ and proves that $\phi^{-1}$ is continuous at 0 .

Since $\phi$ is bijective it follows from (3) that $\phi^{-1}$ is a subadditive bijection of $\mathbb{R}_{+}$. In view of Theorem 1 the function $\phi^{-1}$ is a homeomorphism of $\mathbb{R}_{+}$ and, consequently, increasing.

Hence for all $s, t \geq 0$ with $s \leq t$, the numbers

$$
x_{1}:=\phi^{-1}(s), \quad x_{2}:=\phi^{-1}\left(\frac{t-s}{2}\right),
$$

$$
y_{1}:=\phi^{-1}\left(\frac{s+t}{2}\right)-\phi^{-1}(s), \quad y_{2}:=0
$$

are nonnegative. Inserting them in (1) we obtain

$$
\frac{\phi^{-1}(s)+\phi^{-1}(t)}{2} \leq \phi^{-1}\left(\frac{s+t}{2}\right), \quad s, t \geq 0
$$

i.e. $\phi^{-1}$ is Jensen concave. Since $\phi^{-1}$ is continuous and increasing it follows that $\phi$ is convex. This completes the proof.

Re mark 1 . If $f: \mathbb{R}_{+} \rightarrow \mathbb{R}_{+}$satisfies $f(s)+f(t) \leq f(s+t), s, t \geq 0$, then obviously it is increasing and $f(0)=0$. Thus every superadditive bijection of $\mathbb{R}_{+}$is a homeomorphism. This permits us to prove a dual counterpart of Theorem 2.

TheOREM 3. Let $\phi: \mathbb{R}_{+} \rightarrow \mathbb{R}_{+}$be an arbitrary bijection. If $\mathbf{p}_{\phi}$ is superadditive in $\mathbb{R}_{+}^{2}$ then $\phi$ is a concave homeomorphism of $\mathbb{R}_{+}$.

Pr o of. Suppose that $\mathbf{p}_{\phi}$ is superadditive in $\mathbb{R}_{+}^{2}$. It is easy to see that $\phi(0)=0$ and $\phi^{-1}$ is superadditive. By Remark $1, \phi^{-1}$ is a homeomorphism 
of $\mathbb{R}_{+}$. Substituting in the reversed inequality (1) the numbers $x_{1}, x_{2}, y_{1}$, $y_{2}$ defined by (6) we conclude that $\phi$ is concave.

This is a partial converse of Mulholland's inequality stating that if $\phi$ and $\log \circ \phi \circ \exp$ are concave then $\mathbf{p}_{\phi}$ is superadditive [6].

2. An application to a partial converse of Minkowski's inequality for $\mathbf{l}_{2}^{p}$-norm. Mulholland's inequality provides us with a broad class of bijective functions $\phi$ for which the functional $\mathbf{p}_{\phi}$ is subadditive in $\mathbb{R}_{+}^{2}$. It is easy to verify that every power function $\phi(t):=\phi(1) t^{p}, p \geq 1$, belongs to this class and that the subadditivity of $\mathbf{p}_{\phi}$ becomes the simplest version of Minkowski's inequality for the $\mathbf{l}_{2}^{p}$-norm. The main result of this section says that, under only some regularity assumptions, the subadditivity of the functional $\mathbf{p}_{\phi}$ implies that $\phi$ is a power function. We start with the following

THEOREM 4. Let $\phi: \mathbb{R}_{+} \rightarrow \mathbb{R}_{+}$be an arbitrary bijection and suppose that the functional $\mathbf{p}_{\phi}$ is subadditive in $\mathbb{R}_{+}^{2}$. Then $\phi$ is a convex homeomorphism of $\mathbb{R}_{+}$and the limit

$$
c_{0}:=\lim _{t \rightarrow \infty} \frac{\phi(t)}{t}
$$

exists where $0<c_{0} \leq \infty$. Moreover:

(a) if $c_{0}<\infty$ then $\phi(t)=c_{0} t(t \geq 0)$;

(b) if $c_{0}=\infty$ and there exists a $p>1$ such that the limit

$$
c:=\lim _{t \rightarrow \infty} \frac{\phi(t)}{t^{p}}
$$

exists, $0<c<\infty$, and the function $\phi^{1 / p}$ is convex then $\phi(t)=c t^{p}(t \geq 0)$.

Proof. By Theorem 2 the function $\phi$ is a convex homeomorphism of $\mathbb{R}_{+}$. Thus $\phi(0)=0$ and, consequently, the function $(0, \infty) \ni t \rightarrow \phi(t) / t$ is increasing. This implies that the limit $c_{0}$ exists and $0<c_{0} \leq \infty$.

Inequality (1) holds by assumptions. Setting $x_{1}=x_{2}:=s, y_{1}=y_{2}:=t$ in (1) we get inequality (2), which means that $f:=\phi^{-1} \circ(2 \phi)$ is subadditive. Since $f$ is increasing it follows that $f^{n}$, the $n$th iterate of $f$, is subadditive, i.e.

$$
\phi^{-1}\left[2^{n} \phi(s+t)\right] \leq \phi^{-1}\left[2^{n} \phi(s)\right]+\phi^{-1}\left[2^{n} \phi(t)\right], \quad s, t \geq 0, n \in \mathbb{N} .
$$

Suppose now that $c_{0}$ is finite. Then $\lim _{t \rightarrow \infty} \phi^{-1}(t) / t=c_{0}^{-1}$. Writing (7) in the form

$$
\frac{\phi^{-1}\left[2^{n} \phi(s+t)\right]}{2^{n} \phi(s+t)} \phi(s+t) \leq \frac{\phi^{-1}\left[2^{n} \phi(s)\right]}{2^{n} \phi(s)} \phi(s)+\frac{\phi^{-1}\left[2^{n} \phi(t)\right]}{2^{n} \phi(t)} \phi(t)
$$

for $s, t>0, n \in \mathbb{N}$, and letting $n$ tend to infinity we hence get

$$
\phi(s+t) \leq \phi(s)+\phi(t), \quad s, t>0,
$$


i.e. $\phi$ is subadditive. On the other hand, $\phi$, being convex and satisfying $\phi(0)=0$, is superadditive. Thus $\phi$ is additive and, consequently, $\phi(t)=c_{0} t$ $(t \geq 0)$ (cf. J. Aczél [1], p. 34, Theorem 1).

Suppose that $c_{0}=\infty$. Now $\lim _{t \rightarrow \infty} \phi^{-1}(t) / t^{1 / p}=c^{-1 / p}$. Therefore writing (7) in the form

$\frac{\phi^{-1}\left[2^{n} \phi(s+t)\right]}{\left[2^{n} \phi(s+t)\right]^{1 / p}}[\phi(s+t)]^{1 / p} \leq \frac{\phi^{-1}\left[2^{n} \phi(s)\right]}{\left[2^{n} \phi(s)\right]^{1 / p}}[\phi(s)]^{1 / p}+\frac{\phi^{-1}\left[2^{n} \phi(t)\right]}{\left[2^{n} \phi(t)\right]^{1 / p}}[\phi(t)]^{1 / p}$ for all $s, t>0, n \in \mathbb{N}$, and letting $n$ tend to infinity we get

$$
[\phi(s+t)]^{1 / p} \leq[\phi(s)]^{1 / p}+[\phi(t)]^{1 / p}, \quad s, t \geq 0,
$$

i.e. $\phi^{1 / p}$ is subadditive. Since $\phi^{1 / p}$ is increasing and by assumption convex, it follows that it is superadditive. Thus $\phi^{1 / p}$ is additive and, consequently, linear. This concludes the proof.

Remark 2. One can easily verify that the assumption of convexity of $\phi^{1 / p}$ in Theorem 4(b) can be replaced by each of the following conditions:

(i) $t \rightarrow \phi\left(t^{1 / p}\right)$ is convex in $(0, \infty)$;

(ii) $t \rightarrow t^{-1} \phi\left(t^{1 / p}\right)$ is increasing in $(0, \infty)$;

(iii) $t \rightarrow t^{-1}[\phi(t)]^{1 / p}$ is increasing in $(0, \infty)$;

(iv) $t \rightarrow \phi\left(t^{1 / p}\right)$ is superadditive in $(0, \infty)$;

(v) $\phi^{1 / p}$ is superadditive.

This improves our earlier result (cf. [4], Corollary 2) where it is assumed that $\phi(0)=0$ and $\phi^{-1}$ is continuous at 0 .

If we drop the convexity assumption in Theorem 4(b) we can only assert that $\phi(t) \geq c t^{p}(t \geq 0)$. In fact, in view of the first part of the proof of Theorem 4(b), where we do not use the convexity assumption, $\phi^{1 / p}$ is subadditive. Therefore

$$
[\phi(n t)]^{1 / p} \leq n[\phi(t)]^{1 / p}, \quad t>0, n \in \mathbb{N},
$$

or, equivalently,

$$
\frac{\phi(n t)}{(n t)^{p}} t^{p} \leq \phi(t), \quad t>0, n \in \mathbb{N} .
$$

As $n$ tends to infinity we hence obtain $c t^{p} \leq \phi(t)$ for all $t>0$ (cf. also [4], Proposition 5). In this connection consider the following

ExAmple. For $p>1$ the function $\phi(t):=t^{p}+t^{p-1}, t \geq 0$, is obviously convex. After some simple calculations we have

$$
(\log \circ \phi \circ \exp )^{\prime \prime}(u)=(u+1)^{-2}, \quad u \in \mathbb{R},
$$

which proves that $\log \circ \phi \circ \exp$ is also convex. Thus, by Mulholland's inequality, $\mathbf{p}_{\phi}$ is subadditive in $\mathbb{R}_{+}^{2}$. Since $\phi$ is not a power function, this 
example shows that in Theorem 4(b) the convexity of $\phi^{1 / p}$ (or of any of its substitutes (i)-(v)) is essential.

Remark 3. It is easy to observe that the assumption of the existence of $p>1$ such that the limit

$$
c:=\lim _{t \rightarrow \infty} \frac{\phi(t)}{t^{p}}
$$

is positive and finite in Theorem 4(b) can be replaced by the existence of a finite positive limit

$$
\lim _{t \rightarrow 0} \frac{\phi(t)}{t^{p}} .
$$

R e m a r k 4. Making use of Remark 3 one can easily get the counterparts of the above results for $\mathbf{p}_{\phi}$ superadditive.

3. Functions conjugate to linear functions and subadditivity. In the proofs of Theorems 2 and 4 we considered functions of the form $f:=\phi^{-1} \circ(a \phi)$, with $a=2^{n}, n \in \mathbb{N}$. Since $\phi \circ f \circ \phi^{-1}(t)=a t$, the function $f$ is $\phi$-conjugate to the linear function $t \rightarrow a t$. In this section we are concerned with some properties of such functions.

Theorem 5. If $\phi: \mathbb{R}_{+} \rightarrow \mathbb{R}_{+}$is a bijection such that

(i) $\phi^{-1}$ is subadditive;

(ii) $\phi^{-1} \circ(a \phi)$ is continuous for some $a>0, a \neq 1$;

(iii) $\phi^{-1}$ is bounded in $(0, c)$ for some $c>0$,

then $\phi$ is a homeomorphism of $\mathbb{R}_{+}$.

Proof. Since $t=0$ is the only fixed point of the homeomorphism $f:=\phi^{-1} \circ(a \phi)$, we have either

(a) $f(t)<t$ for all $t>0$, or

(b) $f(t)>t$ for all $t>0$.

Take an arbitrary $x_{0}>0$ and define

$$
x_{k+1}:=f\left(x_{k}\right) \quad(k=0, \pm 1, \pm 2, \ldots) .
$$

One can easily verify that

$$
\phi\left(x_{k}\right)=a^{k} \phi\left(x_{0}\right) \quad(k=0, \pm 1, \pm 2, \ldots) .
$$

In the sequel, without any loss of generality, we may assume that $a>1$. (In fact, if $0<a<1$, consider the function $\phi^{-1} \circ\left(a^{-1} \phi\right)$ which, being the inverse of $f$, is also a homeomorphism of $\mathbb{R}_{+}$.)

Note that case (a) cannot occur. Indeed, in this case we have $x_{k+1}<x_{k}$ for all integer $k$. Consequently, the limit

$$
c:=\lim _{k \rightarrow \infty} x_{-k}>x_{0}>0
$$

exists. If $c<\infty$ then, by the continuity of $f$, we would have 


$$
f(c)=\lim _{k \rightarrow \infty} f\left(x_{-k}\right)=\lim _{k \rightarrow \infty} x_{-k+1}=c,
$$

which is a contradiction.

Suppose that $c=\infty$ and put $y_{k}:=\phi\left(x_{-k}\right), k \in \mathbb{N}$. Since

$$
\lim _{k \rightarrow \infty} y_{k}=\lim _{k \rightarrow \infty} \phi\left(x_{-k}\right)=\lim _{k \rightarrow \infty} a^{-k} \phi\left(x_{0}\right)=0,
$$

we hence get

$$
\lim _{k \rightarrow \infty} \phi^{-1}\left(y_{k}\right)=\lim _{k \rightarrow \infty} x_{-k}=c=\infty .
$$

This is a contradiction as $\phi^{-1}$ is bounded in a neighbourhood of 0 .

This proves that $f$ satisfies inequality (b). Consequently,

$$
x_{k}<x_{k+1}, \quad k=0, \pm 1, \pm 2, \ldots,
$$

and, by the continuity of $f$,

$$
\lim _{k \rightarrow \infty} f\left(x_{-k}\right)=0, \quad \lim _{k \rightarrow \infty} f\left(x_{k}\right)=\infty .
$$

It follows that

$$
(0, \infty)=\bigcup_{k=-\infty}^{\infty}\left[x_{k}, x_{k+1}\right) .
$$

Because $\left[x_{k}, x_{k+1}\right)=f\left(\left[x_{k-1}, x_{k}\right)\right)$, we have $\phi\left(\left[x_{k}, x_{k+1}\right)\right)=a \phi\left(\left[x_{k-1}, x_{k}\right)\right)$. Setting

$$
E_{k}:=\phi\left(\left[x_{k}, x_{k+1}\right)\right) \quad(k=0, \pm 1, \pm 2, \ldots),
$$

we obtain

$$
E_{k}=a E_{k-1}:=\left\{a t: t \in E_{k-1}\right\}=a^{k} E_{0} \quad(k=0, \pm 1, \pm 2, \ldots) .
$$

Since $\phi$ is bijective, the sets $E_{k}$ are pairwise disjoint and

$$
(0, \infty)=\bigcup_{k=-\infty}^{\infty} E_{k}
$$

Observe that 0 is not a limit point of the set $E_{0}$. For an indirect proof suppose that this is not the case. Then there exists a sequence $y_{k} \in E_{0}$ $(k \in \mathbb{N})$ such that $z_{k}:=a^{k} y_{k}(k \in \mathbb{N})$ satisfies $\lim _{k \rightarrow \infty} z_{k}=0$. Since $z_{k} \in E_{k}$ $(k \in \mathbb{N})$, we hence get $\phi^{-1}\left(z_{k}\right) \in\left[x_{k}, x_{k+1}\right)(k \in \mathbb{N})$ and $\lim _{k \rightarrow \infty} \phi^{-1}\left(z_{k}\right)$ $=\infty$. This contradicts the boundedness of $\phi^{-1}$ in a neighbourhood of 0 .

Hence, putting $\delta_{k}:=\inf E_{k}(k=0, \pm 1, \pm 2, \ldots)$, we have

$$
\delta_{k}=a^{k} \delta_{0}>0 \quad(k=0, \pm 1, \pm 2, \ldots) .
$$

Taking an arbitrary integer $n$ we get 


$$
\begin{aligned}
\phi\left(\left[x_{n}, \infty\right)\right) & =\phi\left(\bigcup_{k=n}^{\infty}\left[x_{k}, x_{k+1}\right)\right)=\bigcup_{k=n}^{\infty} \phi\left(\left[x_{k}, x_{k+1}\right)\right) \\
& =\bigcup_{k=n}^{\infty} E_{k} \subseteq \bigcup_{k=n}^{\infty}\left[\delta_{k}, \infty\right)=\left[\delta_{n}, \infty\right)=\left[a^{n} \delta_{0}, \infty\right)
\end{aligned}
$$

which means that for every $t$,

$$
0<t<a^{n} \delta_{0} \Rightarrow \phi^{-1}(t)<x_{n} .
$$

Since $n=0, \pm 1, \pm 2, \ldots$ is arbitrary and $\lim _{n \rightarrow \infty} x_{-n}=0$, this implies that $\phi^{-1}$ is continuous at 0 . Now Theorem 1 concludes the proof.

ExAMPLE. Let $\alpha: \mathbb{R} \rightarrow \mathbb{R}$ be a discontinuous additive involution (cf. [2], p. 293, Theorem 2) and let $\phi:=|\alpha|_{\mid \mathbb{R}_{+}}$(the restriction of $|\alpha|$ to $\mathbb{R}_{+}$). Since $\alpha=\alpha^{-1}$, it is easy to verify that

$1^{\mathrm{o}} \phi^{-1}$ and $\phi$ are subadditive bijections of $\mathbb{R}_{+}$;

$2^{\circ}$ for every rational $a>0$ the function $\phi^{-1} \circ(a \phi)$ is linear;

$3^{\circ}$ the graphs of $\phi$ and $\phi^{-1}$ are dense in $\mathbb{R}_{+}^{2}$.

Thus $\phi^{-1} \circ(a \phi)$ can be homeomorphic (even linear) for extremely irregular $\phi$. This also shows that assumption (iii) of Theorem 5 is essential.

The main result of this section reads as follows.

THEOREM 6. If a bijective function $\phi: \mathbb{R}_{+} \rightarrow \mathbb{R}_{+}$satisfies the following conditions:

(i) $\phi^{-1}$ is subadditive;

(ii) there exists $n \in \mathbb{N}, n>1$, such that $\phi^{-1} \circ(n \phi)$ is subadditive;

(iii) $\phi^{-1}$ is bounded in a neighbourhood of 0 ,

then $\phi$ is a homeomorphism of $\mathbb{R}_{+}$.

Proof. Clearly $f:=\phi^{-1} \circ(n \phi)$ is a bijection of $\mathbb{R}_{+}$. From (i) we get

$$
f(t)=\phi^{-1}(n \phi(t)) \leq n \phi^{-1}(\phi(t))=n t, \quad t \in[0, \infty),
$$

which proves that $f$ is continuous at 0 . In view of Theorem 1 the function $f$ is a homeomorphism of $\mathbb{R}_{+}$. Now Theorem 5 concludes the proof.

Remark 5. The function $\phi: \mathbb{R}_{+} \rightarrow \mathbb{R}_{+}$given by $\phi(t):=t^{-1}(t>0)$ and $\phi(0)=0$ is a subadditive bijection of $\mathbb{R}_{+}$. Moreover, for every $a>0$ the function $\phi^{-1} \circ(a \phi)(t)=a t(t \geq 0)$ is additive. This shows that the assumption (iii) of Theorem 6 is indispensable.

4. A contribution to the converse of Minkowski's integral inequality. For a measure space $(\Omega, \Sigma, \mu)$ denote by $\mathbb{S}=\mathbb{S}(\Omega, \Sigma, \mu)$ the set of all $\mu$-integrable step functions $x: \Omega \rightarrow \mathbb{R}$ and by $\mathbb{S}_{+}$the set of all nonnegative $x \in \mathbb{S}$. It can be easily verified that for every bijection $\phi: \mathbb{R}_{+} \rightarrow \mathbb{R}_{+}$ 
such that $\phi(0)=0$ the functional $\mathbf{P}_{\phi}: \mathbb{S} \rightarrow \mathbb{R}_{+}$given by the formula

$$
\mathbf{P}_{\phi}(x):=\phi^{-1}\left(\int_{\Omega} \phi \circ|x| d \mu\right), \quad x \in \mathbb{S},
$$

is well-defined. In [3] the following converse of Minkowski's inequality has been proved.

If $(\Omega, \Sigma, \mu)$ is a measure space with two sets $A, B \in \Sigma$ such that

$$
0<\mu(A)<1<\mu(B)<\infty
$$

and $\phi$ is a bijection such that $\phi(0)=0, \phi^{-1}$ is continuous at 0 and

$$
\mathbf{P}_{\phi}(x+y) \leq \mathbf{P}_{\phi}(x)+\mathbf{P}_{\phi}(y), \quad x, y \in \mathbb{S}_{+},
$$

then $\phi(t)=\phi(1) t^{p}(t \geq 0)$ for some $p \geq 1$.

At least from the aesthetic point of view this result would be more satisfactory if the purely technical continuity assumption could be dropped. This seems to be a rather difficult question. To explain the role of this assumption observe that $\phi(0)=0$ and the triangle inequality for $\mathbf{P}_{\phi}$ imply that $f:=\phi^{-1}$ is subadditive in $\mathbb{R}_{+}$. Therefore, by Theorem $1, \phi$ is a homeomorphism of $\mathbb{R}_{+}$. This is a starting point of the proof given in [3].

An attempt at replacing the continuity of $\phi^{-1}$ at 0 by the boundedness of $\phi^{-1}$ in a neighbourhood of 0 causes serious difficulties (cf. [5]). However, making use of Theorem 6 , we can prove the following

TheOREM 7. Let $(\Omega, \Sigma, \mu)$ be a measure space with two disjoint sets $A, B \in \Sigma$ such that $\mu(A) \in(0,1)$ and $\mu(B) \in \mathbb{N} \backslash\{1\}$. If $\phi: \mathbb{R}_{+} \rightarrow \mathbb{R}_{+}$is an arbitrary bijection such that $\phi(0)=0$, the function $\phi^{-1}$ is bounded in a neighbourhood of 0 and

$$
\mathbf{P}_{\phi}(x+y) \leq \mathbf{P}_{\phi}(x)+\mathbf{P}_{\phi}(y), \quad x, y \in \mathbb{S}_{+},
$$

then $\phi(t)=\phi(1) t^{p}(t \geq 0)$ for some $p \geq 1$.

Proof. Put $a:=\mu(A), n:=\mu(B)$ and denote by $\chi_{C}$ the characteristic function of a set $C$. Setting in (8),

$$
x:=x_{1} \chi_{A}+x_{2} \chi_{B}, \quad y:=y_{1} \chi_{A}+y_{2} \chi_{B} \quad\left(x_{1}, x_{2}, y_{1}, y_{2} \geq 0\right),
$$

we get the inequality

$$
\begin{aligned}
\phi^{-1}\left[a \phi\left(x_{1}+y_{1}\right)+\right. & \left.n \phi\left(x_{2}+y_{2}\right)\right] \\
& \leq \phi^{-1}\left[a \phi\left(x_{1}\right)+n \phi\left(x_{2}\right)\right]+\phi^{-1}\left[a \phi\left(y_{1}\right)+n \phi\left(y_{2}\right)\right]
\end{aligned}
$$

for all nonnegative $x_{1}, x_{2}, y_{1}, y_{2}$. Hence, specifying these variables in an obvious way and making use of the assumption $\phi(0)=0$, we infer that $\phi^{-1}$ and $\phi^{-1} \circ(n \phi)$ are subadditive. Since $\phi^{-1}$ is bounded in a neighbourhood of 0 , Theorem 6 implies that $\phi$ is a homeomorphism of $\mathbb{R}_{+}$. We also have $0<\mu(A)<1<\mu(B)<\infty$. Now our theorem results from the converse of Minkowski's inequality quoted above. 
We end this paper with one more application of Theorem 2. Strengthening slightly the assumptions on the underlying measure space, we prove a converse of Minkowski's inequality without any regularity assumptions.

TheOREM 8. Let $(\Omega, \Sigma, \mu)$ be a measure space with three sets $A, B, C$ $\in \Sigma$ such that

$$
0<\mu(A)<1, \quad \mu(B)=\mu(C)=1, \quad B \cap C=\emptyset .
$$

If $\phi: \mathbb{R}_{+} \rightarrow \mathbb{R}_{+}$is a bijection such that $\phi(0)=0$ and

$$
\mathbf{P}_{\phi}(x+y) \leq \mathbf{P}_{\phi}(x)+\mathbf{P}_{\phi}(y), \quad x, y \in \mathbb{S}_{+},
$$

then $\phi(t)=\phi(1) t^{p}(t \geq 0)$ for some $p \geq 1$.

Proof. One can easily verify that taking in the triangle inequality (8),

$$
x:=x_{1} \chi_{B}+x_{2} \chi_{C}, \quad y:=y_{1} \chi_{B}+y_{2} \chi_{C} \quad\left(x_{1}, x_{2}, y_{1}, y_{2} \geq 0\right),
$$

we get inequality (1). It means that the functional $\mathbf{p}_{\phi}: \mathbb{R}_{+}^{2} \rightarrow \mathbb{R}_{+}$given by the formula

$$
\mathbf{p}_{\phi}(x):=\phi^{-1}\left[\phi\left(x_{1}\right)+\phi\left(x_{2}\right)\right], \quad x=\left(x_{1}, x_{2}\right),
$$

is subadditive. By Theorem 2 the function $\phi$ is a homeomorphism of $\mathbb{R}_{+}$ and, consequently, $\phi^{-1}$ is continuous at 0 . Since $0<\mu(A)<1$ and $\mu(B \cup$ $C)=2$, our theorem follows from the above quoted converse of Minkowski's inequality.

\section{References}

[1] J. Aczél, Lectures on Functional Equations and Their Applications, Academic Press, New York 1966.

[2] M. Kuczma, An Introduction to the Theory of Functional Equations and Inequalities, Polish Scientific Publishers and Silesian University, Warszawa-Kraków-Katowice 1985.

[3] J. Matkowski, The converse of the Minkowski's inequality theorem and its generalization, Proc. Amer. Math. Soc. 109 (1990), 663-675.

[4] J. Matkowski and T. Świątkowski, Quasi-monotonicity, subadditive bijections of $\mathbb{R}_{+}$, and characterization of $L^{p}$-norm, J. Math. Anal. Appl. 154 (1991), 493-506.

[5] - , - On subadditive functions, Proc. Amer. Math. Soc., to appear.

[6] H. P. Mulholland, On generalizations of Minkowski's inequality in the form of a triangle inequality, Proc. London Math. Soc. 51 (1950), 294-307.

DEPARTMENT OF MATHEMATICS

TECHNICAL UNIVERSITY

WILLOWA 2

43-309 BIELSKO-BIAŁA, POLAND
INSTITUTE OF MATHEMATICS TECHNICAL UNIVERSITY AL. POLITECHNIKI 11 90-924 ŁÓDŹ, POLAND 